\title{
Numerical analysis of the influence of particular autogyro parts on the aerodynamic forces
}

\author{
Zbigniew Czyż ${ }^{1,}$, Pawet Karpiński ${ }^{1}$, Tomasz Lusiak$^{2}$, and Tomasz Szczepanik ${ }^{3}$ \\ ${ }^{1}$ Lublin University of Technology, Faculty of Mechanical Engineering, Department of Thermodynamics, Fluid Mechanics and \\ Aviation Propulsion Systems, Nadbystrzycka 36 Str., 20-618 Lublin, Poland \\ ${ }^{2}$ Polish Air Force Academy, Department of Airframe and Engine, 35 Dywizjonu 303 Str., 08-521 Dęblin, Poland \\ ${ }^{3}$ Institute of Aviation, Aerodynamics Department, Krakowska 110/114, 02-256 Warsaw, Poland
}

\begin{abstract}
This paper describes the research gyrocopter and presents the methodology of numerical calculations for this type of research objects. There are also the results of an ANSYS Fluent three-dimensional simulation of an airflow around a gyrocopter, excluding an airflow around its main rotor and propeller blades. The calculations enabled basic aerodynamic characteristics. The analysis of the numerical calculations focuses on the impact of gyrocopter individual sections like a fuselage, tail, tail boom, etc., on aerodynamic forces and moments, as well as coefficients of aerodynamic forces and moments. The research scope covers an angle of attack $\alpha$ ranging between $-20^{\circ}$ and $25^{\circ}$ at a sideslip angle equal to 0 .
\end{abstract}

\section{Introduction}

Gyrocopters are widely used for sports and leisure activities and they have recently been gaining popularity as they can be used to travel fast short distances, i.e. up to hundreds of kilometres and to perform patrol and rescue missions. Their advantage is undoubtedly their short takeoff and low speed horizontal landing. This type of aircraft is also cheaper to purchase, maintain and operate than small standard-configured helicopters.

There are few publications on research into the gyroplane aerodynamic construction so the publications on helicopters should be referred to if investigating the state of the art. Most of the works on gyroplanes investigates and discusses modelling of a main rotor. Based on the characteristics of the NACA profile, drag acting on the main rotor during the prerotation of a gyrocopter main rotor is defined in [1]. The value of this force was used to calculate the power required to propel the rotor to achieve the assumed speed. Another example of a work that studies aerodynamics of the rotor is [2], where the numerical model to calculate aerodynamic forces and moments acting on the gyrocopter rotor is defined.

The common methods to aerodynamically study the aircraft are computational fluid dynamics (CFD) and wind tunnel experiments. Both were applied in [3] to study drag that occurs on the model of a helicopter fuselage. Particle Image Velocimetry (PIV) was applied in wind tunnel experiments. The validation of both methods was followed by specifying drag coefficients of the fuselage and elements connected to it to estimate interference drag. Furthermore, it has been found that the rear fuselage section is largely responsible for the generated drag. It was shown that the optimisation of the shape leads to a reduced drag coefficient. A numerical analysis of a helicopter fuselage and its wind-tunnel validation is described in [4]. The authors optimised there the mesh of a model of a fuselage and applied a hybrid model of turbulence-detached eddy simulation (DES).

A non-standard approach in an experimental investigation of an airflow around a gyrocopter was followed by the authors in [5]. To investigate how the drag force that generates on a model of a gyrocopter is impacted by speed, a research object was mounted to a car roof. The experimental results were compared with the CFD results and it was found that the numerical analysis differs from real measurements because of the idealconditioned simulation, i.e. steady-state, ideal gas as well as the vehicle-disturbed airflow. To fast study and optimise aerodynamics of a compound gyroplane, methods other than CFD can be applied. They are discussed in [6] where a fast method of aerodynamic calculation (FMAC), combining the advantages of the theory of the blade element moment and the estimation method, is applied.

The authors [7] created their own statisticalengineering-model-based method. Such a model enabled them to define, with the required accuracy, aerodynamic coefficients of a helicopter fuselage for varied angles of attack.

\footnotetext{
* Corresponding author: z.czyz@pollub.pl
} 
Similar studies are discussed in [8] where aerodynamic forces occurs on a standard-configured helicopter fuselage, depending on an angle of attack, a sideslip angle and a side flow.

CFD is applied in [9] to determine drag of a helicopter fuselage according to the Reynolds number. The numerical calculations enabled them to specify the volume of interference drag of a wind tunnel prop with drag of the fuselage.

Aerodynamic forces and moments acting on the aircraft have an impact on its performance and stability. The work [10] investigates gyrocopter flight longitudinal stability. The analysis of the mathematical model and experiments enabled the conclusion that rotor speed is an important parameter for stability. Moreover, a vertical position of centre of gravity in relation to a rotor drag axis may affect longitudinal stability. A preliminary analysis of aerodynamics shall be carried out for the largest aircraft surfaces such as a fuselage or a tail. Components of forces and moments that have extremal values are also important. The fuselage of both helicopter and gyrocopter is the largest part of its entire construction, so its aerodynamic characteristics impacts most on overall aircraft aerodynamics [11].

While investigating the characteristics of coefficients of aerodynamic forces and moments in rotary wing aircraft, the main rotor surface area is usually considered, however, the correlation of reference values of similar aircraft occurs then. To facilitate the comparison of individual types of aircraft, the $f$ parameter, i.e. equivalent flat-plate area is applied, given as [11]:

$$
f=\frac{D}{0,5 \cdot \rho V^{2}}
$$

where D - drag, $\rho$ - air density, V - airflow speed

This paper investigates what portion of total drag, lift and pitching moment is generated on individual elements of the gyrocopter construction, depending on an angle of attack at a constant sideslip angle equal to 0 . Accordingly, a three-dimensional computational model of the aircraft was formed to create an Ansys Fluent tetrahedral mesh, to define boundary conditions, specify a model of turbulence, and finally perform simulations for varied angles of attack. Later, aerodynamic characteristics were created, and conclusions were formulated referring to the obtained results. The impact of the operation of the main rotor and propeller blades on the airflow around the gyrocopter construction was ignored.

\section{Research object and boundary conditions}

The numerical research is based on the Ansys Fluent. Our calculations use the k-SST turbulence model. Aerodynamic forces and moments are specified in relation to the coordinate system associated with the gyrocopter as in Figure 1. It is a right-handed system. The moment along the longitudinal axis $x$ defines roll, along the lateral axis $y$ defines pitch, along the perpendicular axis $z$ defines yaw. It was assumed that a positive moment causes a clockwise rotation around the $\mathrm{z}$ axis, seen from the beginning of the system in the direction of the positive return of the axis. Angle of attack $\alpha$ and sideslip angle $\beta$ are calculated clockwise as positive values.
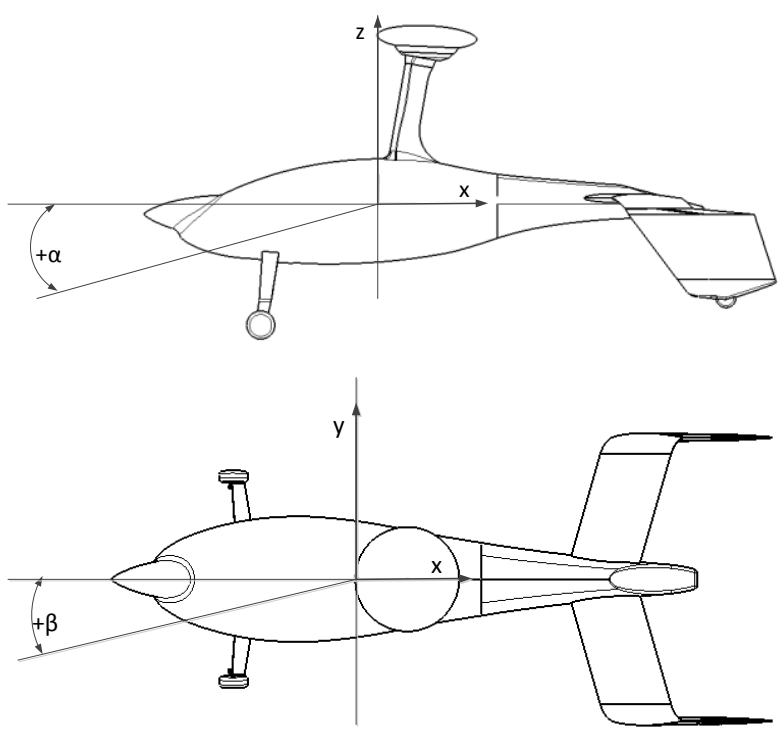

Figure 1. Coordinate system and directions of angles of attack $\alpha$ and a sideslip angle $\beta$ applied in the numerical calculations.

The measuring area of the research object is a cuboid (Figure 2) which walls are $20 \mathrm{~m}$ away from the research object on the $x$ axis, $15 \mathrm{~m}$ on the $y$ axis and $15 \mathrm{~m}$ on the $\mathrm{z}$ axis (at both sides). The gyrocopter is $6,348 \mathrm{~mm} \mathrm{x} 2,802$ $\mathrm{mm} x 3,148 \mathrm{~mm}$ (length $\mathrm{x}$ width $\mathrm{x}$ height).

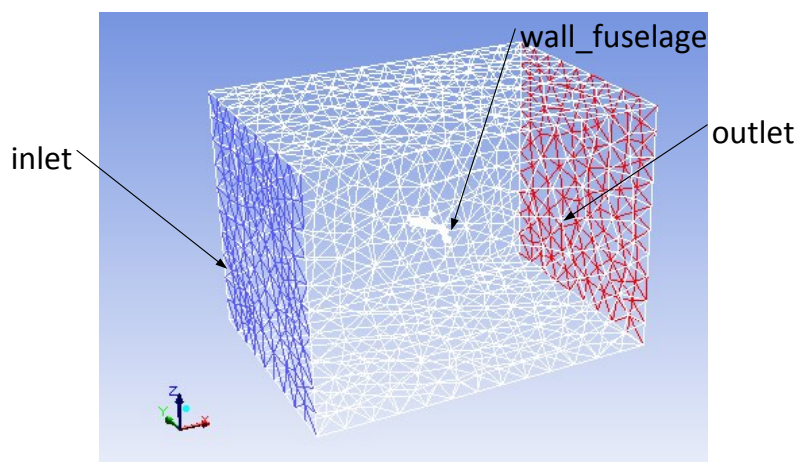

Figure 2. Boundary conditions.

On such a defined calculation domain, there were applied the conditions of velocity inlet and pressure outlet. The remaining walls, including the surfaces of the gyrocopter fuselage are defined as the wall, however, they are distinguished and named basic elements of the gyrocopter construction, i.e. a head, left leg, right leg, fuselage, mast, nose, left vertical stabiliser, right vertical stabiliser, left horizontal stabiliser, right horizontal stabiliser. 


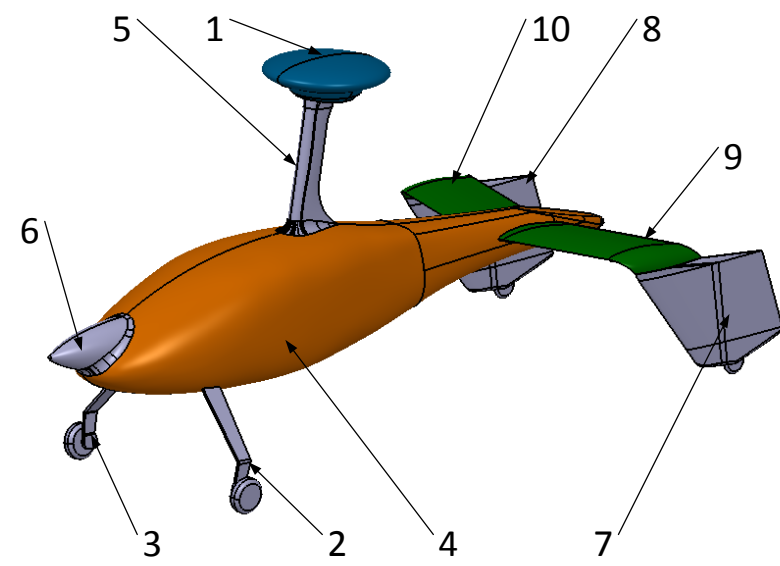

Figure 3. Sections of the gyrocopter construction in the numerical research: 1 - head, 2 - left leg, 3 - right leg, 4 fuselage, 5 - mast, 6 - nose, 7 - vertical stabiliser (left), 8 vertical stabiliser (right), 9 - horizontal stabiliser (left), 10 horizontal stabiliser (right).

The research conditions were pressure-based and steady. Air is a fluid material. A compressible flow was assumed, and a defined material was ideal-gas. In this case, for velocity $\mathrm{v}=100 \mathrm{~km} / \mathrm{h}$ and temperature $\mathrm{T}=288$ $\mathrm{K}$, the boundary speed is $367.4 \mathrm{~km} / \mathrm{h}$ (speed of sound for these conditions is $340 \mathrm{~m} / \mathrm{s}$, i.e. $1,224.6 \mathrm{~km} / \mathrm{h})$. Turbulent intensity is assumed as $1 \%$ and turbulent viscosity ratio as 5 . The basic settings of the numerical research are given in Table 1.

Table 1. General settings of the numerical calculations.

\begin{tabular}{|c|c|c|c|}
\hline General & Type & \multicolumn{2}{|c|}{ Pressure-based } \\
\hline & Time & \multicolumn{2}{|c|}{ Steady } \\
\hline Models & Viscous & \multicolumn{2}{|c|}{ k- $\omega$ (2-eqn) SST } \\
\hline \multirow{4}{*}{ Materials } & $\begin{array}{c}\text { Fluid } \\
\text { materials }\end{array}$ & \multicolumn{2}{|c|}{ Air } \\
\cline { 2 - 4 } & Density & \multicolumn{2}{|c|}{ Ideal-gas } \\
\cline { 2 - 4 } & Viscosity & Constant & $\begin{array}{c}1,7894 \cdot 10^{-5} \\
\mathrm{~kg} / \mathrm{ms}\end{array}$ \\
\hline \multirow{3}{*}{$\begin{array}{c}\text { Boundary } \\
\text { conditions }\end{array}$} & \multirow{2}{*}{ Inlet } & Velocity & $28 \mathrm{~m} / \mathrm{s}$ \\
\cline { 3 - 4 } & & $\begin{array}{c}\text { Turbulent intensity } \\
\text { Turbutio }\end{array}$ & $1 \%$ \\
\hline
\end{tabular}

\section{Research plan and methodology}

The research was performed for the research gyrocopter for angles of attack ranging from $-20^{\circ}$ to $+25^{\circ}$ every $5^{\circ}$ at a constant sideslip angle $\beta=0^{\circ}$. Consequently, there were 10 calculation cases for which were read the values of aerodynamic forces and moments acting on the individual sections of the gyrocopter construction. Then, they were summed up to achieve total aerodynamic forces and moments acting on the whole gyrocopter construction. These, in turn, enabled basic aerodynamic characteristics, i.e. plotted against the correlation of coefficients of aerodynamic forces and moments as a function of an angle of attack $\alpha$. These coefficients were calculated from the following formulas:

$$
C_{i}=\frac{P_{i}}{0,5 \cdot \rho V^{2} \cdot \pi R^{2}} \quad i=x, y, z
$$

$$
C_{m i}=\frac{M_{i}}{0,5 \cdot \rho V^{2} \cdot \pi R^{3}} \quad i=x, y, z
$$

The values of reference parameters applied to determine the above coefficients are in Table 2.

Table 2. Reference values of the parameters.

\begin{tabular}{|c|c|c|c|}
\hline Parameter & Symbol & Value & Unit \\
\hline $\begin{array}{c}\text { Radius of the } \\
\text { main rotor blade }\end{array}$ & $R$ & 5 & $\mathrm{~m}$ \\
\hline Flight velocity & $V$ & 28 & $\mathrm{~m} / \mathrm{s}$ \\
\hline Air density & $\rho$ & 1.226 & $\mathrm{~kg} / \mathrm{m}^{\wedge} 3$ \\
\hline $\begin{array}{c}\text { Ambient } \\
\text { temperature }\end{array}$ & $T$ & 288 & $\mathrm{~K}$ \\
\hline
\end{tabular}

The geometry of the gyrocopter, to be researched, required to be adapted to CFD calculations. The repair of the geometric model, i.e. connecting individual components, complementing certain shortcomings, discontinuity and holes, was done in the CATIA v5. The next step was to import the model into the Design Modeler of the Ansys Workbench. The model was simplified further on, mainly to reduce the number of its fragmented surfaces, to repair them, as well as to remove any acute angles, fragmented edges, etc. The repairing tools were: Repair, Hard Edges, Edges, Sems, Holes, Silvers, Spikes, Faces. The fragmented surfaces were connected with the Merge tool. Having completed any necessary simplifications of the geometry, a fluid domain (i.e. a research area) was created with the Enclosure tool by closing the research object in the cuboid. It was decided to create a universal geometric model, see Figure 4, for different position angles of the gyrocopter.

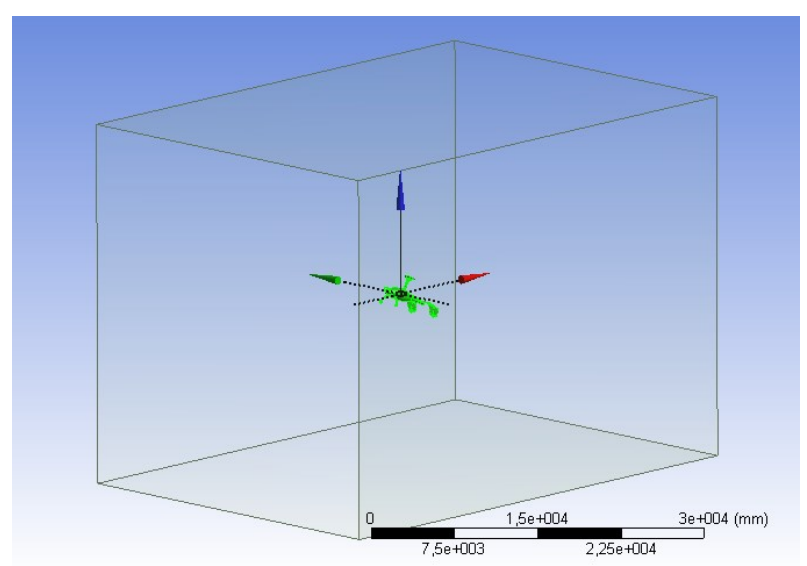

Figure 4. Positioning the model of the gyrocopter in the research area - isometric view.

It consisted in isolating the research object with cylinders, which enabled us to change the position of the gyrocopter in relation to any axis. Defining the angles of attract and sideslip angles was followed by adding the previously isolated cylinders with the embedded shape of the gyrocopter to the rest of the geometry, so that a uniform and coherent shape was created. This method can guarantee that operations, even such as giving a virtual topology, can be recorded at the level of the Ansys/Meshing. While changing, for instance, the angle of 
attack, this program does not lose the location and is still capable of recognising corresponding each other characteristic quantities of the geometry.

The geometries of this type are most often discretised by means of tetrahedral cells. Cell size and quantity and quality can significantly affect calculations, but on the other hand, defining them can facilitate mesh creation. Figure 5 shows the created mesh of the research model of the gyrocopter.

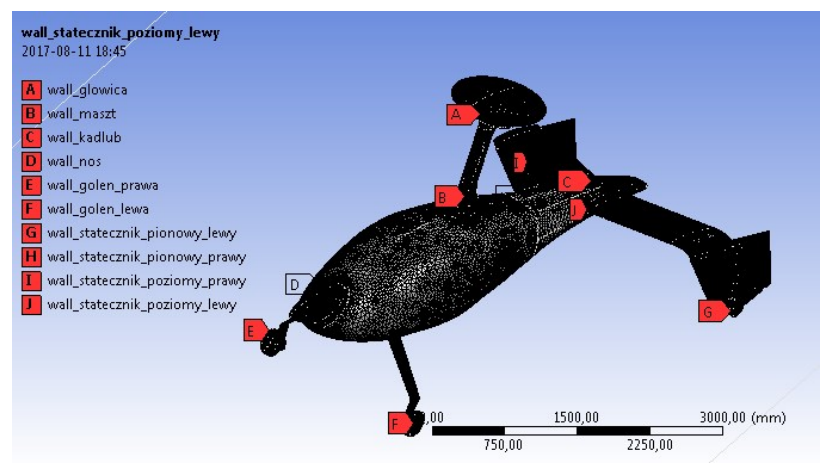

Figure 5. Mesh of the model of the research gyrocopter isometric view.

This is the mesh created by the Tetrahedrons method with an advanced function of the Curvature dimension. The Inflation with the Smooth Transition with 7 layers and a growth rate of 1.15 was applied on the fuselage surface. The mesh was about 6.7 million cells in total.

\section{Results and discussion}

The simulation performed for 10 simulation cases (for varied angles $\alpha$ ) enabled us to achieve the values of the aerodynamic forces and moments acting on the individual sections of the gyrocopter construction. Having summed up these individual values, the values of total forces and moments acting on the whole construction of the gyrocopter were achieved. Table 3 shows the values of the aerodynamic forces and moments, whereas Table 4 the values of the coefficients of aerodynamic forces and moments in relation to each axis for the research range of angles of attack $\alpha$ and a sideslip angle $\beta=0$.

Table 3. Aerodynamic forces and moments in relation to each axis for the research range of angles of attack $\alpha$ and a sideslip angle $\beta=0^{\circ}$.

\begin{tabular}{|c|c|c|c|c|c|c|}
\hline $\left.\boldsymbol{\alpha} \mathbf{(}^{(}\right)$ & $\boldsymbol{P}_{\boldsymbol{x}}(\mathbf{N})$ & $\boldsymbol{P}_{\boldsymbol{y}}(\mathbf{N})$ & $\boldsymbol{P}_{\boldsymbol{z}}(\mathbf{N})$ & $\boldsymbol{M}_{\boldsymbol{x}}(\mathbf{N m})$ & $\left.\boldsymbol{M}_{\boldsymbol{y}} \mathbf{( N m}\right)$ & $\boldsymbol{M}_{\boldsymbol{z}}(\mathbf{N m})$ \\
\hline-20 & 544,72 & 0,55 & $-995,55$ & 3,10 & 934,68 & $-0,33$ \\
\hline-15 & 412,34 & 3,63 & $-943,98$ & $-3,49$ & 1049,45 & $-4,87$ \\
\hline-10 & 273,70 & 3,60 & $-783,02$ & $-3,19$ & 913,80 & $-0,59$ \\
\hline-5 & 208,04 & 1,59 & $-383,78$ & $-0,60$ & 418,48 & $-0,01$ \\
\hline 0 & 184,74 & $-3,28$ & 47,67 & 1,71 & $-100,71$ & $-1,24$ \\
\hline 5 & 198,14 & 2,55 & 450,85 & $-5,39$ & $-611,08$ & $-0,91$ \\
\hline 10 & 244,13 & 2,50 & 776,25 & $-3,33$ & $-1121,29$ & $-0,45$ \\
\hline 15 & 365,93 & 0,84 & 956,04 & $-4,18$ & $-1279,95$ & $-11,60$ \\
\hline 20 & 509,58 & 3,53 & 1101,11 & $-4,13$ & $-1421,13$ & $-3,96$ \\
\hline 25 & 660,29 & 1,53 & 1208,53 & $-3,94$ & $-1493,59$ & $-1,44$ \\
\hline
\end{tabular}

Table 4. Values of the coefficients of the aerodynamic forces and moments in relation to each axis for the research range of angles of attack $\alpha$ and a slip angle $\beta=0^{\circ}$.

\begin{tabular}{|c|c|c|c|c|c|c|}
\hline $\left.\boldsymbol{\alpha} \mathbf{(}^{\circ}\right)$ & $\boldsymbol{C}_{\boldsymbol{x}}(-)$ & $\boldsymbol{C}_{\boldsymbol{y}}(-)$ & $\boldsymbol{C}_{\boldsymbol{z}}(-)$ & $\boldsymbol{C}_{\boldsymbol{m} \boldsymbol{x}}(-)$ & $\boldsymbol{C}_{\boldsymbol{m} \boldsymbol{y}}(-)$ & $\boldsymbol{C}_{\boldsymbol{m} \boldsymbol{z}}(-)$ \\
\hline-20 & 0,01444 & 0,00001 & $-0,02639$ & 0,00002 & 0,00496 & 0,00000 \\
\hline-15 & 0,01093 & 0,00010 & $-0,02502$ & $-0,00002$ & 0,00556 & $-0,00003$ \\
\hline-10 & 0,00725 & 0,00010 & $-0,02076$ & $-0,00002$ & 0,00484 & 0,00000 \\
\hline-5 & 0,00551 & 0,00004 & $-0,01017$ & 0,00000 & 0,00222 & 0,00000 \\
\hline 0 & 0,00490 & $-0,00009$ & 0,00126 & 0,00001 & $-0,00053$ & $-0,00001$ \\
\hline 5 & 0,00525 & 0,00007 & 0,01195 & $-0,00003$ & $-0,00324$ & 0,00000 \\
\hline 10 & 0,00647 & 0,00007 & 0,02058 & $-0,00002$ & $-0,00594$ & 0,00000 \\
\hline 15 & 0,00970 & 0,00002 & 0,02534 & $-0,00002$ & $-0,00679$ & $-0,00006$ \\
\hline 20 & 0,01351 & 0,00009 & 0,02919 & $-0,00002$ & $-0,00753$ & $-0,00002$ \\
\hline 25 & 0,01750 & 0,00004 & 0,03203 & $-0,00002$ & $-0,00792$ & $-0,00001$ \\
\hline
\end{tabular}

Due to numerous results, the values of the forces and moments acting on the individual sections of the gyrocopter for all the cases (for each of the angle of attack $\alpha$ ) are not given. Figures 6 and 7 show the forces acting on the individual sections of the gyrocopter for angles of attack $\alpha=-20^{\circ}, \alpha=0^{\circ} \alpha=25^{\circ}$ and a slip angle $\beta=0^{\circ}$. Similarly, Figure 8 shows pitching moment $M y$ acting on the individual sections of the gyrocopter for angles of attack $\alpha=-20^{\circ}, \alpha=0^{\circ}, \alpha=25^{\circ}$ and a slip angle $\beta=0^{\circ}$.

The values of the lateral force $P y$, as well as rolling moment $M x$ and yawing moment $M z$ are not given. Their values are negligible due to the symmetry of the gyrocopter construction and the symmetry of the airflow (sideslip angle $\beta=0^{\circ}$ ) - the forces and moments generated on each half balance each other out. The values of drag $P x$ and lift $P z$ are meaningful in terms of aircraft performance, and the value of pitching moment $M y$ has an impact on aircraft longitudinal stability.

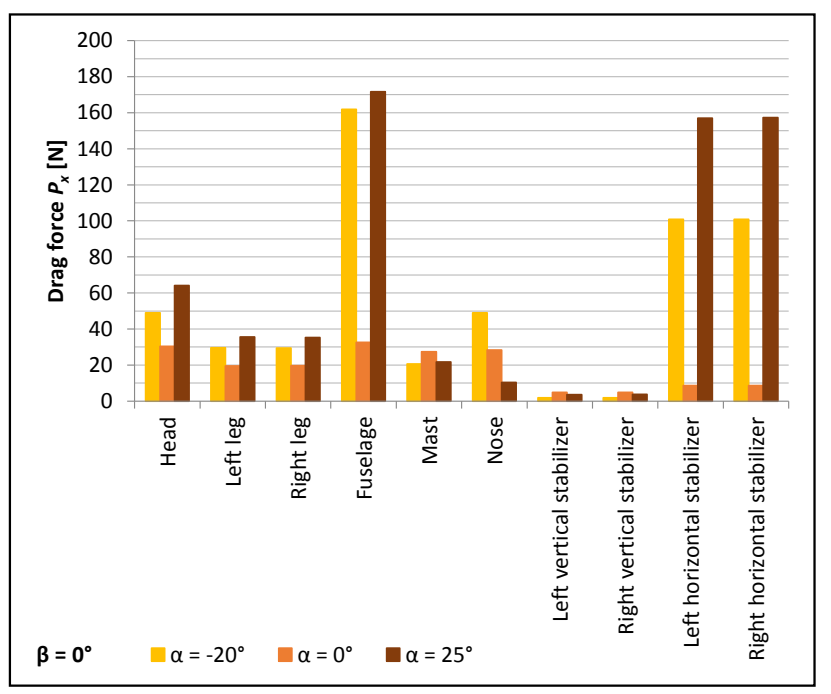

Figure 6. Drag Px acting on the individual sections of the gyrocopter for angles of attack $\alpha=-20^{\circ}, \alpha=0^{\circ}, \alpha=25^{\circ}$ and a sideslip angle $\beta=0^{\circ}$.

The research shows that at the angle of attack equal to 0 , the sections like the head, fuselage, mast and nose (at least $15 \%$ share) have the largest share in the generation of drag $P x$; the legs also have a significant share, i.e. more than $10 \%$. For large and small angles of attack, the share of drag force, generated on the fuselage and horizontal stabilisers, increases a lot. The force generated on the legs and head also increases. 


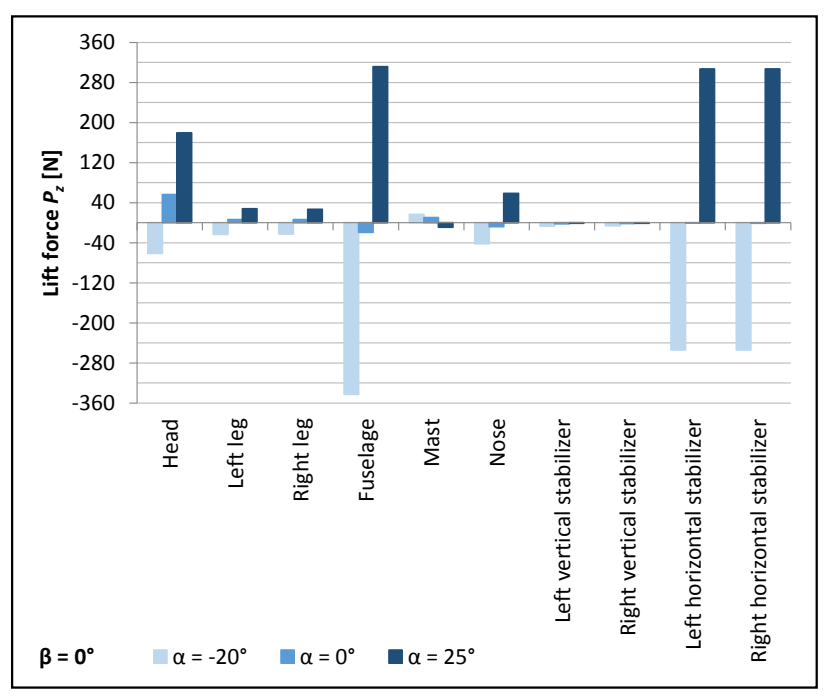

Figure 7. Drag $P z$ acting on the individual sections of the gyrocopter for angles of attack $\alpha=-20^{\circ}, \alpha=0^{\circ}, \alpha=25^{\circ}$ and a sideslip angle $\beta=0^{\circ}$.

At the angle of attack equal to 0 , the largest positive lift $P z$ is generated on the gyrocopter head. The mast and legs also show a small share. In the case of a large negative angle of attack, a large negative lift force is generated on the fuselage and horizontal stabilisers. The forces generated on the head, legs and nose are negative. For a large angle of attack, the forces generated on the fuselage, horizontal stabilisers, nose and legs are positive. The forces generated on the head, nose and horizontal stabilisers significantly increase.

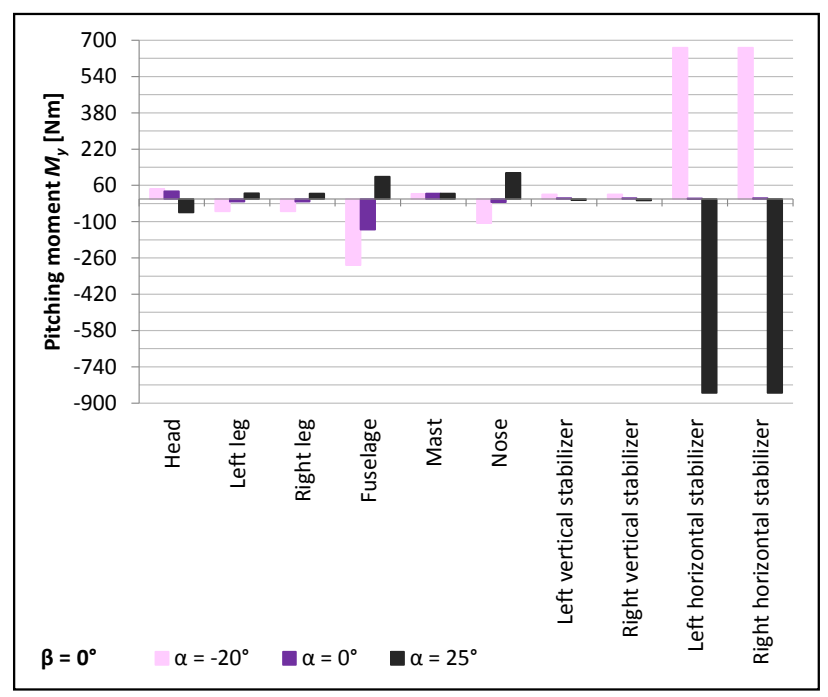

Figure 8. Pitching moment $M y$ acting on the individual sections of the gyrocopter for angles of attack $\alpha=-20^{\circ}, \alpha=0^{\circ}, \alpha=25^{\circ}$ and a sideslip angle $\beta=0^{\circ}$.

For the angle of attack equal to 0 , the pitching moment $M y$ is negative on the fuselage, nose and legs. Its largest absolute value is generated on the fuselage. Highly positive pitching moments are generated on the head and mast. For a large negative angle of attack, the pitching moments generated on the fuselage, head and nose increase their negative values. Positive pitching moments on horizontal stabilisers rapidly increase. For a large positive angle of attack, a large positive pitching moment is generated on horizontal stabilisers. The moments on the fuselage, nose and legs change their values into positive, whereas the ones on the legs, horizontal and vertical stabilisers change into negative.

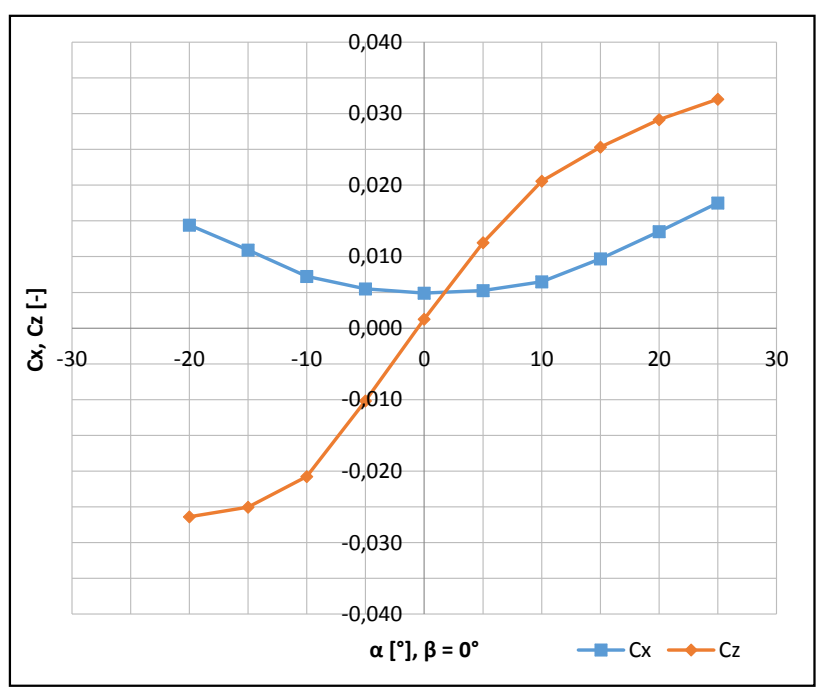

Figure 9. Drag coefficient $C x$ and lift coefficient $C z$ as a function of an angle of attack $\alpha$ for a sideslip angle $\beta=0^{\circ}$.

Drag coefficient $C x$ is the lowest for the angle of attack equal to 0 . With increasing and decreasing the angle of attack, this coefficient increases to achieve its maximum value at an angle $\alpha=25^{\circ}$. Lift coefficient $C z$ reaches 0 at the angle of attack of approx. $0.5^{\circ}$. This coefficient changes approximately linearly within the angles of attack ranging from $-10^{\circ}$ to $10^{\circ}$.

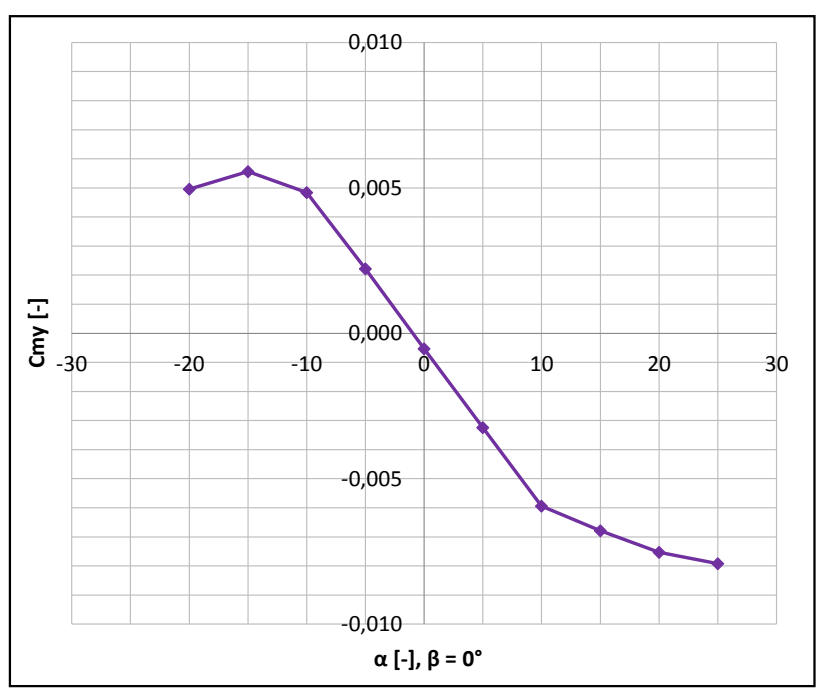

Figure 10. Coefficient of aerodynamic pitching moment $C m y$ as a function of an angle of attack $\alpha$ for a sideslip angle $\beta=0^{\circ}$.

Coefficient of aerodynamic pitching moment Cmy changes linearly in the angles of attack from $-10^{\circ}$ to $10^{\circ}$. Its highest value is at $\alpha=-15^{\circ}$, whereas the lowest one is at $\alpha=25^{\circ}$. One of the criteria of aircraft longitudinal stability is that a derivative from a coefficient of aerodynamic pitching moment after an angle, i.e. $\mathrm{d} C_{m y} / \mathrm{d} \alpha<0$ should be negative (a descending function). In the range of angles 
between $-10^{\circ}$ and $10^{\circ}$, this derivative is constant as -0.0005 so the gyroplane can maintain longitudinal stability.

\section{Conclusions}

The paper shows the results of the numerical simulation of the airflow around the model of the gyrocopter for different angles of attack and a constant sideslip angle equal to 0 . The research focused on how individual sections of the construction of the gyroplane, i.e. its head, left and right legs, fuselage, mast, nose, left and right vertical stabilisers, left and right horizontal stabilisers are impacted by the generated aerodynamic forces and moments. There were calculated coefficients of aerodynamic forces and moments to create basic aerodynamic characteristics.

This work has been financed under Grant Agreement No. POIR.01.01.01-00-0056/16

\section{References}

1. Z. Czyż, T. Łusiak, D. Czyż, D. Kasperek, Adv. Sci. Technol. Res. J., 10(31), 169 (2016)

2. B. McCormick, AIAA 2002-5950, 1 (2002)

3. A. Batrakov, L. Garipova, A. Kusyumov, S. Mikhailov, J. AIRCRAFT, 52(5), 1634 (2015)

4. F. Vogel, Ch. Breitsamter, N. Adams, NNFM, 112, 603 (2010)

5. Z. Czyż, P. Magryta, M. Szlachetka, Adv. Sci. Technol. Res. J., 9(26), 89 (2015)

6. T. Ma, S. Hao, P. Xue, G. Li, W. Gan, ICMEIS 2015, Adv. Eng. Res., 91 (2015)

7. H. Sheikhi, A. Saghaie, Chin. J. of Aeronaut., 30(1), 175 (2017)

8. A. Filippone, Aeronaut. J., 111(1117), 175 (2007)

9. V. Gleize, AIAA, 2001(0999), 1 (2001)

10. S. Houston, J. Guid. Control. Dynam., 21(3), 391 (1998)

11. J.G. Leishman, Principles of helicopter aerodynamics (Cambridge University Press 2006) 